\title{
Revascularização do miocárdio sem circulação extracorpórea em pacientes multiarteriais: experiência de 250 casos
}

\author{
Bruno Botelho PINHEIRO*, Walter Vosgrau FAGUNDES*, Maria Cardoso RAMOS**, Vera Lúcia B. \\ AZEVEDO**, Jânio Moreira SILVA** \\ *Do Serviço de Cirurgia Cardiovascular do grupo Clinicord - Hospital Santa Genoveva, Goiânia. **Do Serviço de Cardiologia \\ do grupo Clinicord - Hospital Santa Genoveva, Goiânia.
}

RBCCV 44205-596

\begin{abstract}
Resumo
Método: No período de agosto de 1997 a maio de 2001, 250 pacientes multiarteriais, consecutivos, foram submetidos à cirurgia de revascularização do miocárdio sem circulação extracorpórea. A faixa etária dos pacientes variou de 38 a 83 anos (média de 59,9 anos), dos quais $62 \%$ eram do sexo masculino. A principal indicação cirúrgica foi a insuficiência coronariana crônica $(82 \%)$. Todos os pacientes foram operados por esternotomia mediana.

Resultados: Três $(1,2 \%)$ pacientes necessitaram de instalação de circulação extracorpórea. Nos demais 247 pacientes, realizou-se 592 anastomoses, com uma média de 2,4 pontes/ paciente. A artéria torácica interna esquerda foi utilizada em $198(80,1 \%)$ pacientes, a artéria torácica interna direita em 5 (2\%) pacientes e a veia safena em $247(100 \%)$ pacientes. As artérias coronárias mais revascularizadas foram o ramo interventricular anterior (89\%) e o ramo marginal esquerdo (53\%). A mortalidade hospitalar global foi de $4 \%$, sendo a principal causa o infarto pós-operatório $(1,2 \%)$. Morbidade pósoperatória foi constatada em $23(9,3 \%)$ pacientes. O tempo médio de permanência hospitalar foi de 7,7 dias.

Conclusão: Conclui-se da presente investigação, que a revascularização do miocárdio sem circulação extracorpórea em pacientes multiarteriais é factível, reprodutível e com baixo índice de complicações pós-operatórias.
\end{abstract}

Descritores: Revascularização miocárdica, métodos. Isquemia miocárdica, cirurgia. Circulação extracorpórea.

\section{INTRODUÇÃO}

A cirurgia de revascularização do miocárdio (RM) com utilização de circulação extracorpórea (CEC), embora seja considerada como "padrão ouro" no tratamento da insuficiência coronariana multiarterial, tem sido ultimamente bastante questionada devido aos efeitos deletérios da $\mathrm{CEC}^{(1-4)}$.

\section{Abstract}

Methods: From August 1997 to May 2001, off-pump coronary artery bypass grafting (OPCAB) was performed in 250 consecutive patients with multivessel disease. Ages ranged from 38 to 83 years (mean-age 59.9 years) and $62 \%$ of the patients were males. The main surgical indication was chronic coronary insufficiency $(82 \%)$. The surgical approach was through median sternotomy in all patients.

Results: Three (1.2\%) patients needed cardiopulmonary bypass support. In the 247 remaining patients, we performed 592 grafts (mean 2.4 grafts/patient). The left internal thoracic artery was used in $198(80.1 \%)$ patients, the right internal thoracic artery in $5(2 \%)$ patients and the saphenous vein in all patients $(100 \%)$. The most frequently revascularized coronary arteries were the anterior interventricular branch $(89 \%)$ and the obtuse marginal $(53 \%)$. The mortality rate was $4 \%$ and the main primary cause of death was myocardial infarction $(1.2 \%)$. Twenty-three $(9.3 \%)$ patients had major complications in the postoperative period. The mean hospital stay was 7.7 days.

Conclusion: In conclusion, OPCAB can be safely performed in patients with multivessel disease, with low rates of postoperative complications.

Descriptors: myocardial revascularization, surgery. Myocardial Ischemia, surgery. Extracorporeal circulation.

Desta forma, a revascularização do miocárdio com o coração batendo, inicialmente realizada por Kolessov, em 1967, na União Soviética ${ }^{(5)}$, renasceu como uma alternativa bastante atrativa para o tratamento cirúrgico da insuficiência coronariana; sendo popularizada por vários autores nas décadas de 70 e $80^{(6-9)}$.

Para ser qualificada como procedimento alternativo eficaz

Trabalho realizado no grupo Clinicord - Hospital Santa Genoveva. Goiânia, GO, Brasil. Recebido para publicação em agosto de 2001. Endereço para correspondência: Bruno Botelho Pinheiro. Av. República do Líbano, 411. Goiânia, GO, Brasil. CEP 74070-040. E-mail: bbpmt@cultura.com.br 
em relação ao convencional, a RM com coração batendo deve ser reprodutível em qualquer tipo de anatomia coronariana, utilizar enxertos vasculares semelhantes, apresentar resultados técnicos equivalentes, taxa de morbidade e mortalidade comparáveis, baixo índice de conversão para CEC e, principalmente, possibilitar uma revascularização completa do miocárdio, visto que, isso representa um dos maiores benefícios para o paciente coronariopata $^{(10)}$.

Vários autores têm excluído ou não sistematizado a revascularização do miocárdio sem CEC em pacientes multiarteriais, que necessitam de abordagem dos ramos marginais esquerdos e ventriculares posteriores da artéria circunflexa $^{(11-13)}$.

O objetivo do nosso trabalho é demonstrar a nossa experiência e a sistematização da cirurgia de revascularização do miocárdio sem CEC em pacientes multiarteriais.

\section{MÉTODO}

No período de agosto de 1997 a maio de 2001, todos os pacientes $(\mathrm{pt})$ portadores de insuficiência coronariana multiarterial com indicação de revascularização cirúrgica do miocárdio (258 casos), encaminhados ao nosso serviço, foram primariamente elegíveis para se submeterem ao procedimento sem a utilização de circulação extracorpórea, com a finalidade de verificarmos a exeqüibilidade do método. Após explicação detalhada do caráter investigativo do mesmo, obtivemos por escrito consentimento pósinformado dos participantes. Como critérios de exclusão adotamos: choque cardiogênico (4 pt), fração de ejeção < $30 \%$ (2 pt) e não aceitação do método pelo paciente (2 pt).

Portanto, 250 pacientes multiarteriais consecutivos foram submetidos à cirurgia de revascularização do miocárdio sem circulação extracorpórea. Cento e cinquenta e cinco pacientes (62\%) eram do sexo masculino e 95 (38\%), do sexo feminino. A faixa etária dos pacientes variou de 38 a 83 anos, com média de 59,9 anos.

A indicação cirúrgica foi a insuficiência coronariana crônica em 205 (82\%) casos, a angina instável em 40 (16\%), o infarto agudo do miocárdio em $3(1 \%)$ e a isquemia miocárdica silenciosa em $2(1 \%)$. Dezoito $(7,2 \%)$ pacientes apresentavam revascularização do miocárdio prévia, 12 $(4,8 \%)$ já tinham se submetido a angioplastia coronária e 7 $(2,8 \%)$ eram portadores de lesão de tronco de coronária esquerda.

As doenças associadas mais freqüentes foram: hipertensão arterial sistêmica, diabetes mellitus, infarto agudo do miocárdio prévio, doença pulmonar obstrutiva crônica, dislipidemia, acidente vascular cerebral e insuficiência renal crônica (Tabela 1).
Tabela 1. Doenças associadas mais freqüentes

\begin{tabular}{lll}
\hline & $\mathrm{N}$ & $\%$ \\
\hline Hipertensão Arterial Sistêmica & 93 & 38,0 \\
Diabetes Mellitus & 36 & 14,5 \\
Infarto Agudo do Miocárdio & 20 & 8,0 \\
Dislipidemia & 15 & 6,0 \\
DPOC & 11 & 4,4 \\
Insuficiência Renal Crônica & 6 & 2,4 \\
Acidente Vascular Cerebral & 5 & 2,0 \\
\hline
\end{tabular}

\section{Técnica Cirúrgica}

A via de acesso utilizada foi a esternotomia mediana convencional. Os enxertos vasculares foram obtidos através da dissecção da artéria torácica interna esquerda (ATIE), artéria torácica interna direita (ATID) e/ou veia safena (VS). Após abertura do saco pericárdico, realizou-se a anticoagulação com administração de heparina sódica (Liquemine $^{\mathrm{R}}$ ) na dose de $3 \mathrm{mg} / \mathrm{kg}$, mantendo o tempo de coagulação ativado (TCA) maior que 400 segundos. A exposição do coração foi conseguida através da passagem de um ponto de Vicryl n ${ }^{\circ} 1$ (Ethicon, São Paulo, SP, Brasil) na porção central do seio oblíquo do pericárdio posterior e fixado a uma gaze aberta ${ }^{(14)}$. Com a mobilização e tração da gaze pudemos facilmente visualizar todas as artérias coronárias; inclusive as da face posterior do coração.

As artérias coronárias foram imobilizadas com a utilização de estabilizador CTS Ultima OPCAB System ${ }^{\mathrm{R}}$ (Cupertino, CA, USA). Após interrupção temporária do fluxo coronariano, com torniquete proximal de Polipropileno n ${ }^{\circ}$ 4-0 (Ethicon, São Paulo, SP, Brasil), e abertura longitudinal da artéria coronária, introduziu-se "shunt" intracoronário CTS Flocoil (Cupertino, CA, USA) em todos os pacientes (Figura 1).

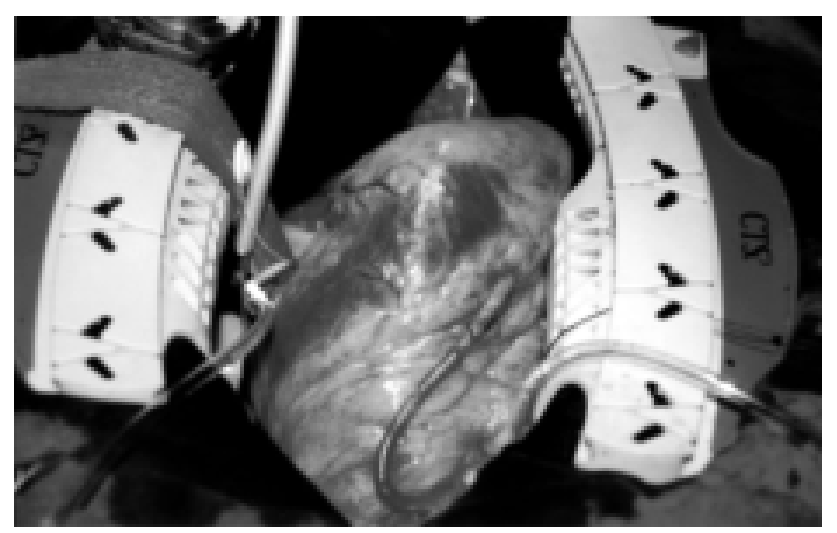

Fig. 1 - Posicionamento do coração para acesso dos ramos da face lateral e inferior, através da tração de uma gaze aberta fixada na porção central do seio oblíquo do pericárdio posterior. Observamos a realização de uma ponte de veia safena para ramo marginal esquerdo, em um paciente de 72 anos, com lesão de tronco de coronária esquerda. 
As anastomoses distais foram realizadas com chuleio contínuo de Polipropileno no 7-0 (Ethicon, São Paulo, SP, Brasil). Não se empregou nenhuma droga cronotrópica negativa com a finalidade de facilitar as anastomoses e nem torniquete distal na artéria coronária.

Os enxertos arteriais (ATIE, ATID) foram sempre utilizados "in situ" e em nenhum caso realizou-se anastomose em "Y" dos mesmos com veia safena. Houve manipulação da aorta ascendente através de clampeamento tangencial em todos os casos. As anastomoses proximais das veias safenas na aorta ascendente foram realizadas com chuleio contínuo de Polipropileno n ${ }^{\circ}$-0 (Ethicon, São Paulo, SP, Brasil).

Sempre revascularizamos em primeiro lugar as artérias ocluídas ou com lesões suboclusivas. Caso não existam tais lesões, utilizamos a seguinte seqüência de tratamento das artérias: circunflexa e seus ramos, ramo diagonalis, ramos diagonais, ramo interventricular anterior, coronária direita e seus ramos. Dois fios de marcapasso epicárdicos são passados antes da manipulação da artéria coronária direita.

A reversão da heparinização foi conseguida através da infusão de cloridrato de protamina na proporção de $0,8: 1,0$ da concentração de heparina sódica. Realizada revisão da hemostasia, drenagem e fechamento por planos.

\section{RESULTADOS}

Ocorreu instabilidade hemodinâmica grave em 3 (1,2\%) pacientes, necessitando a instalação de circulação extracorpórea.

Nos demais 247 pacientes o procedimento transcorreu sem maiores problemas, sendo realizadas 592 anastomoses, com uma média de 2,4 pontes/paciente. A ATIE foi utilizada em $198(80,1 \%)$ pacientes e a ATID em $5(2 \%)$ pacientes. O enxerto de veia safena foi utilizado em todos os casos. As artérias coronárias mais revascularizadas em ordem decrescente de freqüência foram: ramo interventricular anterior (RIA), ramo marginal esquerdo (MgE), ramo diagonal (DI), coronária direita (CD), ramo diagonalis (DG), ramo interventricular posterior (RIP), ramo ventricular posterior (VP) e ramo marginal direito (MgD) (Figura 2).

Fig. 2 - Artérias coronárias revascularizadas

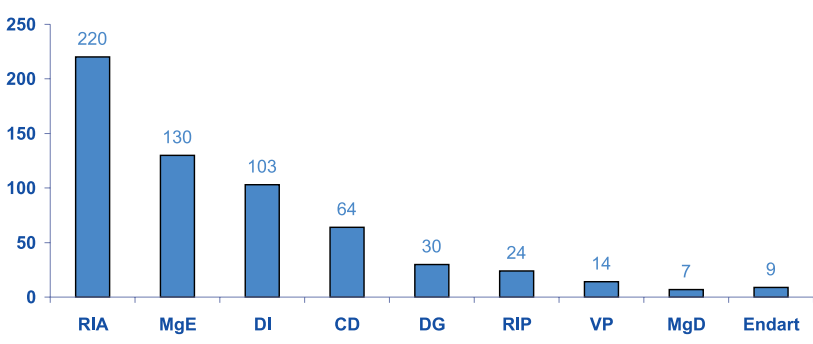

Em $9(3,6 \%)$ pacientes realizou-se endarterectomia de artéria coronária (4 CD, $3 \mathrm{MgE}$ e 2 RIA). Não houve no grupo de 247 pacientes nenhum outro tipo de procedimento cirúrgico associado.

Em 60 (24,2\%) pacientes necessitou-se a instalação de drogas inotrópicas positivas, durante a realização das anastomoses, para manter a pressão arterial média (PAM) = $>60 \mathrm{mmHg}$. A manutenção das mesmas no período pósoperatório ocorreu em $42(17 \%)$ pacientes. Não houve necessidade de instalação de balão intra-aórtico (BIA) em nenhum caso.

O tempo de intubação orotraqueal variou de 0 a $64 \mathrm{~h}$, com uma média de 4,2h. Em cinqüenta e seis $(22,6 \%)$ pacientes foi retirado o tubo orotraqueal na sala de operações. A permanência na UTI variou de 20 a 120h, com uma média de 46,5h.

O sangramento pós-operatório médio foi de: $259 \mathrm{ml} / 6 \mathrm{~h}$, $368 \mathrm{ml} / 12 \mathrm{~h}$ e $483 \mathrm{ml} / 24 \mathrm{~h}$; acarretando uma necessidade de transfusão sangüínea de 1,8 unidades/paciente.

A mortalidade hospitalar global foi de 4\% (Tabela 2).

Tabela 2. Causas de mortalidade hospitalar

\begin{tabular}{lll}
\hline & $\mathrm{N}$ & $\%$ \\
\hline IAM Pós-operatório & 3 & 1,2 \\
Acidente Vascular Cerebral & 2 & 0,8 \\
Arritmias Ventriculares Intratáveis & 2 & 0,8 \\
Disfunção de VE & 1 & 0,4 \\
Infecção Pulmonar + IRA & 1 & 0,4 \\
Mediastinite & 1 & 0,4 \\
TOTAL & 10 & 4,0 \\
\hline
\end{tabular}

Analisando-se separadamente os grupos de pacientes que tinham revascularização do miocárdio prévia (18 pct.) e lesão de tronco de coronária esquerda (7 pct), a mortalidade foi de $1(5,5 \%)$ paciente no primeiro grupo e nenhum $(0 \%)$ no segundo, respectivamente.

As complicações pós-operatórias de maior relevância manifestaram-se em 23 pacientes (Tabela 3 ).

Tabela 3. Morbidade pós-operatória

\begin{tabular}{lll}
\hline & $\mathrm{N}$ & $\%$ \\
\hline Infecções Superficiais & 7 & 2,8 \\
Fibrilação Atrial & 4 & 1,6 \\
Arritmias Ventriculares Sustentadas & 2 & 0,8 \\
Insuficiência Respiratória & 2 & 0,8 \\
Infecção Pulmonar & 2 & 0,8 \\
Confusão Mental & 2 & 0,8 \\
ICC & 1 & 0,4 \\
Trombose Venosa Profunda & 1 & 0,4 \\
Tamponamento Cardíaco & 1 & 0,4 \\
Re-Revascularização (oclusão de ponte) & 1 & 0,4 \\
TOTAL & 23 & 9,3 \\
\hline
\end{tabular}

A permanência hospitalar variou de 5 a 21 dias (média de 7,7 dias). 


\section{COMENTÁRIOS}

A revascularização do miocárdio com circulação extracorpórea é o procedimento padrão para o tratamento cirúrgico da insuficiência coronariana multiarterial, pois apresenta ótimas condições de segurança e reprodutibilidade dos resultados. No entanto, a CEC, ao ativar uma resposta inflamatória sistêmica, contribui de forma decisiva para as complicações pós-operatórias e falência de múltiplos órgãos ${ }^{(1-4)}$. Recentemente, vários grupos têm debatido a possibilidade e vantagem da RM com o coração batendo ${ }^{(11-13,15-17)}$.

A maior dificuldade técnica para realizar anastomoses com o coração batendo, podendo comprometer a patência dos enxertos, é o ponto mais polêmico em relação a revascularização do miocárdio sem CEC. O desenvolvimento tecnológico de estabilizadores, permitindo com que o segmento da artéria coronária a ser tratado permaneça completamente imóvel, foi indispensável para o êxito do procedimento ${ }^{(17)}$. Empregamos em todos os nossos casos o estabilizador CTS Ultima OPCAB System ${ }^{\mathrm{R}}$ (Cupertino, CA, USA).

A exposição da artéria circunflexa e seus ramos, através da luxação do coração com um ponto passado no centro do seio oblíquo do pericárdio posterior, permitiu com que a RM sem CEC pudesse ser adotada em um maior número de pacientes ${ }^{(14)}$. Embora alguns autores relatem comprometimento hemodinâmico durante a luxação do coração ${ }^{(17,18)}$, LIMA et al., realizando avaliação intraoperatória, concluíram que a mobilização máxima do coração não induz instabilidade hemodinâmica ${ }^{(19)}$. Em nossa experiência, apenas $1,2 \%$ dos pacientes tiveram de ser submetidos à circulação extracorpórea devido a instabilidade hemodinâmica grave.

Tivemos que utilizar drogas inotrópicas positivas no período per e pós-operatório em $24,2 \%$ e $17 \%$ dos pacientes, respectivamente. YEATMAN et al. utilizaram drogas inotrópicas positivas no pós-operatório de $12 \%$ dos pacientes submetidos a RM sem CEC, com lesão em tronco de coronária esquerda ${ }^{(20)}$. Já o emprego do BIA tem variado de 1,0 a $2,3 \%$ em RM sem CEC ${ }^{(21,22)}$.

A possibilidade de realizar as anastomoses com o coração batendo, utilizando isquemia regional através do garroteamento da artéria coronária, foi comprovada como sendo procedimento seguro ${ }^{(8,23,24)}$. RIVETTI et al., para manter a irrigação do miocárdio distal à coronária a ser revascularizada, oferecendo maior segurança ao cirurgião, idealizou a utilização de uma derivação intraluminal temporária ${ }^{(12)}$. Todos os nossos pacientes foram operados com o uso de "shunt" intra-coronário e em nenhum momento foi empregado o garroteamento distal da artéria coronária, devido ao risco de lesão endotelial e oclusão coronariana no pós-operatório ${ }^{(25,26)}$.

Embora o tempo de intubação orotraqueal médio tenha sido baixo (4,2h), não tivemos redução na permanência dos pacientes na UTI e nem maior precocidade na alta hospitalar. Cabe salientar dois aspectos que podem ter influenciado nessa evolução - $1^{\circ}$ fixação do objetivo primário da avaliação sobre os fatores de morbimortalidade; $2^{\circ}$ parte da população estudada pertencer a outros domicílios, sem possibilidade de um acompanhamento pós-operatório tipo "home care".

A necessidade média de hemotransfusão foi baixa (1,8 unidades/paciente). NADER et al. demonstraram uma redução média de 2,2 unidades de concentrado de hemácias por paciente submetido a revascularização do miocárdio sem CEC, quando comparados aqueles submetidos a RM $\operatorname{com~CEC~}^{(27)}$.

A mortalidade da revascularização do miocárdio sem circulação extracorpórea tem variado de 1,3 a 4,3\% na maioria dos trabalhos ${ }^{(11-13,15-17,22,28,29)}$. É procedimento seguro, reprodutível e de baixa morbi-mortalidade em pacientes multiarteriais ${ }^{(28,29)}$. Em nosso grupo, a mortalidade hospitalar global foi de $4 \%$ (10 pacientes), tendo como principais causas o infarto agudo do miocárdio $(1,2 \%)$, o acidente vascular cerebral $(0,8 \%)$ e as arritmias ventriculares intratáveis $(0,8 \%)$. A incidência do infarto agudo do miocárdio no pós-operatório de RM sem CEC tem variado de 0,6 a $3,6 \%{ }^{(28,30)}$ e o acidente vascular cerebral, de 0 até $7,1 \%$ em pacientes octogenários ${ }^{(31,32)}$.

Vinte e três $(9,3 \%)$ pacientes apresentaram complicações pós-operatórias, sendo as infecções superficiais $(2,8 \%)$ e a fibrilação atrial $(1,6 \%)$ as etiologias mais frequentes. PUSKAS et al. constataram $12,5 \%$ de fibrilação atrial no pós-operatório de revascularização do miocárdio com coração batendo ${ }^{(21)}$.

Vários pesquisadores têm demonstrado vantagens da RM sem CEC em relação ao procedimento com CEC, principalmente na diminuição das taxas de morbidade pósoperatória - menos distúrbios neurológicos ${ }^{(11,31-33)}$; melhor proteção renal ${ }^{(32,34)}$; menos complicações respiratórias e menor tempo de intubação orotraqueal ${ }^{(11,32)}$; menos $\operatorname{arritmias}^{(11,35)}$; menos síndrome de baixo débito ${ }^{(35)}$; menor sangramento pós-operatório e necessidade de transfusão sangüínea ${ }^{(21,27,32)}$ e menos infecções esternais profundas (21). Conseqüentemente, outro aspecto relevante é a diminuição dos custos financeiros ${ }^{(35,36)}$.

A revascularização do miocárdio com o coração batendo tem se mostrado apropriada em grupos especiais de pacientes multiarteriais: com disfunção ventricular esquerda (Fração de Ejeção < = 30\%) e lesão crítica de tronco de coronária esquerda (TCE) ${ }^{(20,37)}$. Realizamos tal procedimento em 7 pacientes com lesão de TCE, sem nenhum óbito hospitalar.

\section{CONCLUSÃO}

Pode-se concluir da presente investigação que a revascularização do miocárdio sem circulação extracorpórea em pacientes multiarteriais é factível, reprodutível e com baixo índice de complicações pósoperatórias; podendo constituir-se em mais uma opção para o tratamento cirúrgico da insuficiência coronariana. 


\section{REFERÊNCIAS BIBLIOGRÁFICAS}

1. Kirklin JK - Prospects for understanding and eliminating the deleterious effects of cardiopulmonary bypass. Ann Thorac Surg 1991; 51:529-31.

2. Brasil LA, Gomes WJ, Salomão R, Buffolo E - Inflammatory response after myocardial revascularization with or without cardiopulmonary bypass. Ann Thorac Surg 1998; 66: 56-9.

3. Srüber M, Cremer JT, Gohrbandt B et al. - Human cytokine responses to coronary artery bypass grafting with or without cardiopulmonary bypass. Ann Thorac Surg 1999; 68: 1330-5.

4. Ascione R, Lloyd CT, Underwood MJ, Lotto AA, Pitsis AA, Angelini GD - Inflammatory response after coronary revascularization with or without cardiopulmonary bypass. Ann Thorac Surg 2000; 69: 1198-204.

5. Kolessov VL-Mammary artery - coronary artery anastomosis as method of treatment for angina pectoris. J Thorac Cardiovasc Surg 1967; 54: 535-44.

6. Trapp WG \& Bisarya R - Placement of coronary artery bypass graft without pump oxygenator. Ann Thorac Surg 1975; 19: 1-9.

7. Ankeney JL - To use or not to use the pump oxygenator in coronary bypass operations. Ann Thorac Surg 1975; 19: $108-9$.

8. Buffolo E, Andrade JCS, Succi JE et al. - Revascularização direta do miocárdio sem circulação extracorpórea: descrição da técnica e resultados iniciais. Arq Bras Cardiol 1982; 38: 365-73.

9. Benetti FJ - Direct coronary surgery with saphenous vein bypass without either cardiopulmonary bypass or cardiac arrest. J Cardiovasc Surg 1985; 26: 217-22.

10. Bell MR, Gersh BJ, Schaff HV et al. - Effects of completeness of revascularization on long-term outcome of patients with three-vessel disease undergoing coronary artery bypass surgery: a report from the Coronary Artery Surgery Study (CASS) Registry. Circulation 1992; 86:446-57.

11. Buffolo E, Andrade JCS, Branco JNR et al. - Revascularização do miocárdio sem circulação extracorpórea: análise dos resultados em 15 anos de experiência. Rev Bras Cir Cardiovasc 1996; 11: 227-31.

12. Rivetti LA, Gandra SMA, Pinto e Silva AM, Campagnucci VP - Revascularização do miocárdio sem circulação extracorpórea com uso de "shunt" intracardíaco: 12 anos de experiência. Rev Bras Cir Cardiovasc 1997; 12: 226-32.
13. Brasil LA, Mariano JB, Santos FM et al. - Revascularização do miocárdio sem circulação extracorpórea: experiência e resultados iniciais. Rev Bras Cir Cardiovasc 2000; 15: 6-15.

14. Bergsland J, Karamanoukian HL, Soltoski PR, Salerno TA "Single suture" for circumflex exposure in off-pump coronary artery bypass grafting. Ann Thorac Surg 1999; 68: 1428-30.

15. Lobo Filho JG, Dantas MCBR, Rolim JGV et al. - Cirurgia de revascularização completa do miocárdio sem circulação extracorpórea: uma realidade. Rev Bras Cir Cardiovasc 1997; 12: 115-21.

16. Baumgartner FJ, Gheissari A, Capouya ER, Panagiotides GP, Katouzian A, Yokoyama T - Technical aspects of total revascularization in off-pump coronary bypass via sternotomy approach. Ann Thorac Surg 1999; 67:1653-8.

17. Jansen EW, Borst C, Lahpor JR et al. - Coronary artery bypass grafting without cardiopulmonary bypass using the Octopus method: results in the first one hundred patients. $J$ Thorac Cardiovasc Surg 1998; 116: 60-7.

18. Grundeman PF, Borst C, Verlaan CW, Meijburg H, Moues $\mathrm{CM}$, Jansen EW - Exposure of the circumflex branches in the tilted, beating porcine heart: echocardiographic evidence of right ventricular deformation and the effect of right or left heart bypass. J Thorac Cardiovasc Surg 1999; 118: 316-23.

19. Lima RC, Escobar MAS, Della Santa RF et al. - Avaliação hemodinâmica intra-operatória na cirurgia de revascularização do miocárdio sem auxílio da circulação extracorpórea. Rev Bras Cir Cardiovasc 2000; 15: 201-11.

20. Yeatman M, Caputo M, Ascione R, Ciulli F, Angelini GD Off-pump coronary artery bypass for critical left main stem disease: safety, efficacy and outcome. Eur J Cardiothorac Surg 2001; 19: 239-44.

21. Puskas JD, Thourani VH, Marshall JJ et al. - Clinical outcomes, angiographic patency, and resource utilization in 200 consecutive off-pump coronary bypass patients. Ann Thorac Surg 2001; 71: 1477-84.

22. Hernandez F, Cohn WE, Baribeau YR et al. - In-hospital outcomes of off-pump versus on-pump coronary artery bypass procedures: a multicenter experience. Ann Thorac Surg 2001; 72: $1528-34$.

23. Krejca M, Skiba J, Szmagala P, Gburek T, Bochenek A - Cardiac troponin $\mathrm{T}$ release during coronary surgery using intermittent cross-clamp with fibrillation, on-pump and off-pump beating heart. Eur J Cardiothorac Surg 1999; 16: 337-41. 
24. Ascione R, Lloyd CT, Gomes WJ, Caputo M, Bryan AJ, Angelini GD - Beating versus arrested heart revascularization: evaluation of myocardial function in a prospective randomized study. Eur J Cardiothorac Surg 1999; 15: 685-90.

25. Izzat MB, Yim AP, El-Zufari MH - Snaring of a coronary artery causing distal atheroma embolization. Ann Thorac Surg 1998; 66: 1806-8.

26. Okazaki Y, Takarabe K, Murayama J et al. - Coronary endothelial damage during off-pump CABG related to coronaryclamping and gas insufflation. Eur J Cardiothorac Surg 2001; 19: 834-9.

27. Nader ND, Khadra WZ, Reich NT, Bacon DR, Salerno TA, Panos A L - Blood product use in cardiac revascularization: comparison of on- and off-pump techniques. Ann Thorac Surg 1999; 68: 1640-3.

28. Cartier R, Brann S, Dagenais F, Martineau R, Couturier A Systematic off-pump coronary artery revascularization in multivessel disease: experience of three hundred cases. J Thorac Cardiovasc Surg 2000; 119: 221-9.

29. Kshettry VR, Flavin TF, Emery RW, Nicoloff DM, Arom KV, Petersen RJ - Does multivessel, off-pump coronary artery bypass reduce postoperative morbidity? Ann Thorac Surg 2000; 69: 1725-31.

30. Arom KV, Flavin TF, Emery RW, Kshettry VR, Janey PA, Petersen RJ-Safety and efficacy of off-pump coronary artery bypass grafting. Ann Thorac Surg 2000; 69: 704-10.
31. Ricci M, Karamanoukian HL, Abraham R et al. - Stroke in octogenarians undergoing coronary artery surgery with and without cardiopulmonary bypass. Ann Thorac Surg 2000; 69: $1471-5$.

32. Yokoyama T, Baumgartner FJ, Gheissari A, Capouya ER, Panagiotides GP, Declusin RJ - Off-pump versus on-pump coronary bypass in high-risk subgroups. Ann Thorac Surg 2000; 70: 1546-50.

33. Diegeler A, Hirsch R, Schneider F et al. - Neuromonitoring and neurocognitive outcome in off-pump versus conventional coronary bypass operation. Ann Thorac Surg 2000; 69: 1162-6.

34. Ascione R, Lloyd CT, Underwood MJ, Gomes WJ, Angelini GD - On-pump versus off-pump coronary revascularization: evaluation of renal function. Ann Thorac Surg 1999; 68: 493-8.

35. Boyd W D, Desai N D, Del Rizzo D F, Novick R J, McKenzie F N, Menkis A H - Off-pump surgery decreases postoperative complications and resource utilization in the elderly. Ann Thorac Surg 1999; 68: 1490-3.

36. Ascione R, Lloyd C T, Underwood M J, Lotto A A, Pitsis A A, Angelini G D - Economic outcome of off-pump coronary bypass surgery: a prospective randomized study. Ann Thorac Surg 1999; 68: 2237-42.

37. Arom K V, Flavin T F, Emery R W, Kshettry V R, Petersen R J, Janey P A - Is low ejection fraction safe for off-pump coronary bypass operation? Ann Thorac Surg 2000; 70: 1021-5. 